Résumés des conférences et travaux

\title{
Langue et littérature hébraïques médiévales et modernes en occident
}

Jean-Pierre Rothschild

\section{OpenEdition}

\section{Journals}

Édition électronique

URL : https://journals.openedition.org/ashp/969

DOI : 10.4000/ashp.969

ISSN : 1969-6310

Éditeur

Publications de l'École Pratique des Hautes Études

\section{Édition imprimée}

Date de publication : 2 février 2011

Pagination : 30-32

ISSN : 0766-0677

\section{Référence électronique}

Jean-Pierre Rothschild, "Langue et littérature hébraïques médiévales et modernes en occident »,

Annuaire de l'École pratique des hautes études (EPHE), Section des sciences historiques et philologiques

[En ligne], 141 | 2011, mis en ligne le 23 février 2011, consulté le 06 juillet 2021. URL : http://

journals.openedition.org/ashp/969; DOI : https://doi.org/10.4000/ashp.969 


\title{
LANGUE ET LITTÉRATURE HÉBRAÏQUES MÉDIÉVALES ET MODERNES EN OCCIDENT
}

\author{
Directeur d'études : M. Jean-Pierre RothschILD
}

Programme de l'année 2008-2009 : I. R. Nissim de Gérone et son école. - II. Le Livre des éléments d'Isaac Israéli et ses traductions latines.

\section{I. $R$. Nissim de Gérone et son école}

Il était dans la logique des recherches des années passées sur Joseph ben Shem Tov ibn Shem Tov de travailler sur l'une de ses sources, le chef de file le plus revendiqué par l'école au moins informelle de philosophes-théologiens objectants au rationalisme, en Espagne, au XV $v^{\mathrm{e}}$ siècle, dont j'ai montré naguère que Joseph $\mathrm{b}$. Shem Tov était l'un des représentants les plus actifs. Il s'agit d'une famille d'esprit qui s'étend sur quatre générations et qui comprend des auteurs considérables, Nissim de Gérone, le célèbre Hasdaï Crescas, Joseph ben Shem Tov et le non moins célèbre Issac Abrabanel, tous élèves directs les uns des autres. La difficulté d'aborder la pensée du Ran, R. Nissim de Gérone (v. 1320-1380), tient à ce qu'il n'a pas écrit de traité systématique mais, outre son œuvre considérable de commentateur du Talmud que nous ne considérons pas ici (bien qu'elle recèle certainement des éléments ou points de méthode d'intérêt philosophique, la recherche sur ce point ne semble pas avoir été tentée), des sermons sur le Pentateuque dans lesquels ils nous faut dégager les thèmes philosophiques de l'exégèse du détail du texte, à la suite de ses successeurs qui s'y sont référés pour l'argumentation philosophique. Il n'est pas exclu, bien qu'impossible à étayer par des preuves textuelles, que sa position doive quelque chose à ses contemporains latins Guillaume d'Occam et Marsile de Padoue - il en va de même, en Provence, quelque trente ans plus tôt, pour Lévi ben Gerson. Toutefois, le cadre exégétique lui-même favorise le recours aux autorités de sa propre tradition textuelle : en " philosophie politique », dont il passe pour avoir été l'un des introducteurs en hébreu ${ }^{1}$, Moïse ben Naḥman (1194-1270), un siècle avant lui, avait fait usage des virtualités offertes par les textes bibliques : Moïse à Mara (Ex. 15,25), Josué à Sichem (Jos. 24,25) auraient développé des « lois et ordonnances » dictées par les circonstances et autres que celles qui avaient été révélées au Sinaï; à la génération suivante, dans le cadre communal, Salomon ben Aderet avait reconnu aux chefs des communautés la faculté de s'écarter

1. M. Lorberbaum, Politics and the Limits of the Law: Secularizing the Political in Medieval Jewish Thought, Stanford, Stanford University Press, 2001, p. 93-123; A. Ravitzky, Religion and State in Jewish Philosophy. Models of Unity, Division, Collision and Subordination, Jérusalem, The Israel Democracy Institute, 2002, p. 64-66; E. Nachalon, « Structural Models of Religion and State in Jewish and Democratic Political Thought: Inevitable Contradiction? The Challenge for Israel », Touro Law Review, 22 (2006), p. 617-744 (721-739). 
de la Torah à titre provisoire et dans l'intérêt public - Stephen Passamaneck vient de réunir dans une monographie une pluralité de positions rabbiniques de même sens ${ }^{1}$. L'influence latine, si elle a eu lieu, n'aurait ainsi fait que réactiver dans la pensée de R. Nissim des potentialités déjà présentes dans la partie du monde juif qui était la sienne. S'il a connu lui-même l'Éthique à Nicomaque, ce n'a pu être que dans la traduction faite en Provence du Commentaire Moyen d'Averroès.

Si les homélies 8 (sur l'autorité de Moïse, d'après Num. 12,6-8) et 11, sur l'institution judiciaire (d'après Deut. 16,18), ont retenu l'attention des spécialistes de la théorie politique, l'homélie 1, sur le thème apparemment apolitique de Gen. 1,1-3, est l'occasion de notables développements à portée non seulement physique mais aussi sociale (l'exemple, emprunté au droit rabbinique, du respect dû à une communauté quel que soit le caractère de ses membres, en témoigne), à propos de la supériorité du tout par rapport à la somme des parties, non seulement dans l'ordre physique mais aussi dans les collectivités humaines, et à propos de la complémentarité des éléments extrêmes, et donc déficients, qui forment un ensemble qui ne manque de rien. L'homélie 11 assume la politique aristotélicienne avant de lui imposer une sévère limitation : même une association de voleurs a besoin d'une loi, Israël comme les autres et aussi pour une raison supplémentaire : parmi ceux qui sont condamnés, certains le sont par pure justice et d'autres, dans l'intérêt de la cité; la Torah a séparé les deux justices : les juges (šofet tĭm) doivent juger selon la première, la seconde relève de l'ordonnance royale (mis $\left.{ }^{e} w a t h a-m e l e k h\right)$. Il se peut que la Torah ait été plus proche du premier que du second but, puisque pour ce dernier les lois (nīmūsey-y-, du grec vó $\mu \circ \varsigma$ ) des nations suffiraient, et qu'ainsi, elle apparaisse moins propre qu'elles à assurer la conservation de la cité, ce qui n'a pas d'importance puisque le droit du roi y suppléait. Le but spécifique de la Torah s'exprime en termes de šefa', d'épanchement divin, où la métaphore peut être une commodité pas forcément à prendre au pied de la lettre; il s'agit en tout cas d'assurer une proximité du peuple juif avec Dieu bien plutôt que d'assurer l'ordre public. Mais en l'absence de roi, les deux justices sont rendues par les juges (TB Sanh. 79), avant eux par Josué (sens de l'injonction que lui donna Moïse en l'intronisant [Deut. 31,7 et 23]) et avant lui par Moïse (à quoi fait référence Deut. 33,5). Que le principal de la Torah soit le droit de la justice et le secondaire le droit royal explique en quoi les Hébreux fautèrent en demandant un roi en I Sam. 8, alors que c'est l'un des trois commandements qui leur furent adressés au moment d'entrer en Terre Sainte : c'est qu'ils voulurent être d'abord et avant tout sous le régime du droit royal. De plus, les rois sont soumis aux prophètes, les juges ne le sont pas; les rois sont en effet plus exposés à l'orgueil et leur soumission peut aussi (Nahmanide) servir d'exemple. Il est à noter que, chez $\mathrm{R}$. Nissim, la raison de l'inadéquation de la loi divine à régir efficacement les conditions sociales effectives tiendrait à sa perfection même du point de vue de la justice (ainsi, il serait injuste d'appliquer le châtiment dû au criminel à défaut d'avertissement préalable dans les formes avant la perpétration de son crime, ce qui rend la peine de mort à peu près impossible à prononcer). - Le texte a été lu dans l'édition des $D^{e}$ rāšōt ha-Ran, Venise, [5]357/[1596/7].

1. S. M. Passamaneck, Modalities in Medieval Jewish Law for Public Order and Safety, Cincinnati, [Hebrew Union College], 2009. 


\section{Le Livre des éléments d'Isaac Israéli et ses traductions latines}

Bien que moins cité au Moyen Âge que son Livre des définitions, le Livre des éléments d'Isaac Israéli ( $c a$ 855-ca 955) n'en connut pas moins un succès remarquable. On n'en est que plus surpris de constater que sa traduction latine n'a pas fait l'objet d'une édition depuis 1515 (Omnia opera Ysaac..., Lyon, B. Trot, f. 4va-10vb). Nous avions établi il y a quelques années ${ }^{1}$ qu'il en existe deux traductions latines, la deuxième, conservée dans au moins cinq manuscrits, procédant sans doute par révision de la première, qui est connue par au moins onze manuscrits et qui fut éditée dans les Euvres complètes. La situation de ce texte en hébreu est comparable : il a été reconnu au XIX ${ }^{\mathrm{e}}$ siècle qu'il en existait deux traductions hébraïques du XIII ${ }^{\mathrm{e}}$ siècle, mais nul travail de critique textuelle n'a été conduit depuis l'édition de S. Fried, Drohobitz Francfort, 1900, qui éditait la traduction d'Abraham ibn Ḥasdaï en utilisant la seconde traduction, due à Moïse ibn Tibbon, dans l'apparat critique. Il serait d'autant plus intéressant de disposer d'éditions critiques des textes des branches latine et hébraïque, indépendantes l'une de l'autre, de la tradition de cet ouvrage, que l'original arabe en est perdu et pourrait être reconstitué grâce à ce double témoignage. C'est dire, aussi, que même le travail d'édition des traductions subsistantes ne pourrait être mené à bien sans le concours d'un arabisant particulièrement compétent dans la langue scientifique en usage en Afrique du Nord au $\mathrm{X}^{\mathrm{e}}$ siècle; la tâche excède, d'ailleurs, ce que l'on peut espérer traiter dans le seul cadre des conférences car, si le texte est assez bref, il est conservé en hébreu dans vingt et un manuscrits. Nous avons cependant cru pouvoir mener quelques investigations préparatoires, tant sur les textes latins que sur les textes hébreux, qui ont formé la part d'exercices de paléographie et de critique textuelle que comporte chaque année notre conférence.

1. J.-P. Rothschild, «Philosophie (gréco-arabe), "philosophie" de la Loi, d'après les sources juives médiévales, dans la littérature latine : un bilan provisoire », Medioevo, 23 (1997 [1998]), p. 473-513 (476-485). 